\title{
A Comprehensive Method for Detecting Fusion Genes in Paediatric Brain Tumours
}

\author{
AKIHIDE KONDO*, YUZABURO SHIMIZU*, SATOSHI ADACHI, IKUKO OGINO, \\ MARIO SUZUKI, OSAMU AKIYAMA and HAJIME ARAI \\ Department of Neurosurgery, Juntendo University Faculty of Medicine, Tokyo, Japan
}

\begin{abstract}
Background: Fusion genes driving tumourigenesis have drawn the attention of researchers and oncologists. Despite the importance of such molecular alterations, there are no comprehensive reproducible methods for detecting fusion genes. Materials and Methods: Nineteen paediatric brain tumours of five types, namely pilocytic astrocytoma, oligodendroglioma, anaplastic astrocytoma, glioblastoma and, ganglioglioma, were examined to detect fusion genes using a pyrosequencing-based method following RNA isolation, $c D N A$ synthesis and real-time polymerase chain reaction. Results: Our method successfully detected KIAA1549-v-raf murine sarcoma viral oncogene homolog B1 (BRAF) fusion in 14 out of 19 patients suffering from five types of paediatric brain tumours providing information on fusion breakpoints within 2 h. Conclusion: A comprehensive method for detecting fusion genes in paediatric brain tumours was evaluated. This method identified KIAA1549-BRAF fusion variants quickly. Our results may help researchers interested in the role of fusion genes in tumourigenesis.
\end{abstract}

In recent years, molecular biology techniques have modified the classification of tumours (1). Although the majority of tumour classifications have been based on histopathological findings, oncologists are noticing that the characteristics revealed by newer techniques may be more helpful than histopathological features (2) However, the pathogenesis of cancer harbouring fewer genetic mutations such as paediatric cancer remains unclear (3). One hypothesis explaining the

This article is freely accessible online.

*These Authors contributed equally to this study.

Correspondence to: Akihide Kondo, 2-1-1 Hongo, Bunkyo-ku, Tokyo, Japan. Tel: +81 338133111, Fax: +81 356898343, e-mail: knd-aki@juntendo.ac.jp

Key Words: Paediatric brain tumours, BRAF, KIAA1549, fusion gene, pyrosequencing. aetiology of these tumours is the presence of chromosomal rearrangements which lead to gene fusions (4). Fusion genes are thought to cause abnormal activation of adjacent genes (5). Sometimes, even when dealing with the same tumour type, the presence of a fusion gene is an independent prognostic factor (6). Moreover, oncologists usually finetune the treatment strength for tumours in the same pathological subdivision based on the presence or absence of fusion genes (6). Therefore, focussing on fusion genes is important both for treatment and advancing research on this mechanism of tumourigenesis. However, detection of these genes is challenging due to the quality of the samples, which may not provide adequate DNA or RNA purity (3). In addition, fusion variants created by different breakpoints may cause different phenotypes $(7,8)$. Moreover, previous studies have revealed correlations between fusion variants and histopathological diagnoses/prognoses (9).

Despite the established importance of fusion genes and their breakpoint variants, no comprehensive method exists for their identification. Moreover, some identification techniques are restricted to molecular biology laboratories and not widely utilized.

The most accurate identification method involves direct sequencing followed by real-time polymerase chain reaction (RT-PCR). However, this method requires s good specimen quality for analysis and is costly, despite developments in DNA and RNA purification techniques (10). Therefore, the most common method for detecting the presence of fusion genes is dual-probed fluorescence in situ hybridization (FISH) (11), although this method does not allow for identification of the fusion breakpoints and has a relatively high rate of false-positive results (11). It is fair to say that no comprehensive, accurate and cost-effective method exists for detection of fusion genes.

The Pyromark system (Qiagen, Hilden, Germany) has been developed as a method to obtain the genetic sequence of a particular site. This method can be used for shorter sequences than direct sequencing methods, but the technique has been shown to be accurate and reproducible enough to detect fusion genes (12). 
Table I. Primer list for detecting three variants of the KIAA1549-v-raf murine sarcoma viral oncogene homolog B1 (BRAF) fusion.

\begin{tabular}{|c|c|c|c|c|}
\hline Assay & Fusion gene & Primers & Sequence (5' to 3') & PCR Product (bp) \\
\hline \multirow[t]{4}{*}{$15-9$} & \multirow[t]{4}{*}{ KIAA1549 exon 15-BRAF exon 9} & Forward & acagacagcgatggcaccta & \multirow[t]{4}{*}{106} \\
\hline & & Reverse (biotinylated) & atcctccatcaccacgaaatc & \\
\hline & & Sequence & actcagcc tacatcgg & \\
\hline & & Dispensation order & GATGACCAGATCTGATGAGAGTAC & \\
\hline \multirow[t]{4}{*}{$16-9$} & \multirow[t]{4}{*}{ KIAA1549 exon 16-BRAF exon 9} & Forward & cagtgggggtccttctacagc (1) & \multirow[t]{4}{*}{89} \\
\hline & & Reverse (biotinylated) & cctccatcaccacgaaatcc & \\
\hline & & Sequence & agacggccaa caatc $(2)$ & \\
\hline & & Dispensation order & GCTGCTAGTGTACTGATCAGAGACTA & \\
\hline \multirow[t]{4}{*}{$16-11$} & \multirow[t]{4}{*}{ KIAA1549 exon 16-BRAF exon 11} & Forward & cagtgggggtccttctacagc (1) & \multirow[t]{4}{*}{97} \\
\hline & & Reverse (biotinylated) & caggaatctcccaatcatcac & \\
\hline & & Sequence & agacggccaa caatc (2) & \\
\hline & & Dispensation order & ACTGCAGTCAACACTAGTAGCACGGACT & \\
\hline
\end{tabular}

Chromosomal rearrangements caused by fusion genes are common in brain tumours (13-15); the KIAA1549 exon-15v-raf murine sarcoma viral oncogene homolog $\mathrm{B} 1(B R A F)$ exon-9 (KIAA1549-BRAF) fusion, is considered a driver of genetic events and a diagnostic marker (13), particularly in pilocytic astrocytoma, a major type of paediatric brain tumour (15). Studies have revealed that specific breakpoints of KIAA1549-BRAF correlate with tumour characteristics of different types of brain tumour (14). Therefore, the detection of a fusion gene by FISH alone is insufficient to reveal its clinical relevance.

In this study, we focused on evaluating the Pyromark system to identify fusion genes and their breakpoints in paediatric brain tumour samples. The time requirements for this method were also assessed and the meaning of our findings for paediatric brain tumours was discussed.

\section{Materials and Methods}

Study design. KIAA1549-BRAF fusions including their fusion breakpoints in paediatric brain tumours were identified using a pyrosequecing-based method.

Tumour samples. Tumour samples were obtained from tumour bulks removed from paediatric patients suffering from brain tumours those were under the age of 15 at the time of diagnosis. Tumour specimens removed from patients during surgery were immediately placed in an RNA-stabilising agent (RNA later; ThermoFisher, Waltham, MA, USA) after collection. The Juntendo University Ethics Committee approved the study (IRB \#2010-014), and written informed consent was obtained from all the patients or their legal guardians. No any exclusion criteria were used for this study.

RNA isolation and cDNA synthesis. Total RNA was prepared from $20 \mathrm{mg}$ of tumour tissue and isolated using All Prep DNA/RNA Mini Kit (Qiagen, Manchester, UK), according to the manufacturer's instructions. The RNA concentration was measured using QuantiFluor RNA System (Promega Corp., Madison, WI, USA) on
Table II. Summary data of the tumour types and fusion positivity.

\begin{tabular}{lcc}
\hline Tumour type & $\begin{array}{c}\text { Number } \\
(\%)\end{array}$ & $\begin{array}{c}\text { Rate of KIAA1549- } \\
\text { BRAF fusion }\end{array}$ \\
\hline Pilocytic astrocytoma & $10(52.6)$ & $90 \%(9 / 10)$ \\
Glioblastoma & $3(15.8)$ & $33 \%(1 / 3)$ \\
Oligodendroglioma & $2(10.5)$ & $100 \%(2 / 2)$ \\
Anaplastic astrocytoma & $2(10.5)$ & $50 \%(1 / 2)$ \\
Ganglioglioma & $2(10.5)$ & $50 \%(1 / 2)$ \\
Total & 19 & $14 / 19(74 \%)$ \\
\hline
\end{tabular}

$B R A F$ : v-Raf murine sarcoma viral oncogene homolog B1.

Quantus $^{\mathrm{TM}}$ Fluorometer (Promega Corp.). A total of 250 ng RNA was reverse transcribed to obtain cDNA using SuperScript ${ }^{\mathrm{TM}}$ IV VILO тм Master Mix (ThermoFisher), following the manufacturer's instructions. RNA extraction required $15 \mathrm{~min}$ and cDNA synthesis $25 \mathrm{~min}$.

KIAA1549-BRAF transcripts by RT-PCR. Three well-documented fusion genes: KIAA1549 exon-15-BRAF exon-9, KIAA1549 exon-16$B R A F$ exon-9 and KIAA1549 exon-16-BRAF exon-11. Three PCRs were performed to detect the three different fusion products of KIAA1549-BRAF using $1 \mu \mathrm{l}(25 \mathrm{pmol} / \mu \mathrm{l})$ of cDNA primers (Table I) and Fast Cycling PCR Kit (Qiagen), following the manufacturer's instructions. PCR was performed with 30 amplification cycles at an annealing temperature of $60^{\circ} \mathrm{C}$. A total of $25 \mu$ l PCR products were obtained in approximately $30 \mathrm{~min}$. The PCR products were then analysed using electrophoresis on $2 \%$ agarose gels.

Pyrosequencing reaction and assay design. Pyrosequencing was performed using $10 \mu \mathrm{l}$ of the PCR product, sequencing primer (Table I) and PyroGold Reagents (Qiagen), according to the manufacturer's instructions, on a PyroMarkQ24 (Qiagen). Pyrogram outputs were analysed using PyroMark Q24 2.0 software (Qiagen) using the short quantification assay. It took approximately $45 \mathrm{~min}$ for this process. 
Table III. Pathological data and fusion breakpoints by case.

\begin{tabular}{|c|c|c|c|c|c|c|c|c|}
\hline \multirow[b]{2}{*}{ Case } & \multirow[b]{2}{*}{ Age at surgery } & \multirow[b]{2}{*}{ Gender } & \multirow[b]{2}{*}{ Diagnosis } & \multirow[b]{2}{*}{ Tumour location } & \multicolumn{4}{|c|}{ KIAA1549-BRAF fusion } \\
\hline & & & & & Detected & ex $15-e x 9$ & ex16-ex9 & ex16-ex11 \\
\hline 1 & 3 Years 5 months & $\mathrm{F}$ & $\mathrm{PA}$ & Optic pathway & Yes & + & & \\
\hline 2 & 4 Years 6 months & M & PA & Optic pathway & Yes & + & & \\
\hline 3 & 5 Years 10 months & $\mathrm{F}$ & PA & Optic pathway & Yes & + & & \\
\hline 4 & 4 Months & $\mathrm{F}$ & PA & Optic pathway & Yes & + & & \\
\hline 5 & 7 Months & $\mathrm{F}$ & PA & Optic pathway & Yes & + & & \\
\hline 6 & 5 Years 10 months & M & PA & Cerebellum & Yes & + & + & \\
\hline 7 & 8 Years 9 months & $\mathrm{F}$ & PA & Cerebellum & Yes & + & + & \\
\hline 8 & 13 Years 11 months & M & PA & Cerebellum & Yes & & + & \\
\hline 9 & 4 Years 7 months & $\mathrm{F}$ & PA & Cerebellum & Yes & + & & + \\
\hline 1011 Years 9 months & $\mathrm{M}$ & PA & Cerebellum & No & & & & \\
\hline 1113 Years 8 months & $\mathrm{F}$ & GBM & Thalamus & Yes & + & + & & \\
\hline 1210 Years & $\mathrm{F}$ & GBM & Thalamus & No & & & & \\
\hline 1312 Years 4 months & $\mathrm{F}$ & GBM & Temporal lobe & No & & & & \\
\hline 149 Years 4 months & $\mathrm{F}$ & OD & Temporal lobe & Yes & + & & & \\
\hline 1511 Years 2 months & M & OD & Parietal lobe & Yes & + & & + & \\
\hline 161 Month & M & AA & Temporal lobe & Yes & + & & & \\
\hline 174 Years 5 months & M & AA & Thalamus & No & & & & \\
\hline 181 Year 9 months & M & GG & Temporal lobe & Yes & + & & & \\
\hline 1914 Years & M & GG & Temporal lobe & No & & & & \\
\hline
\end{tabular}

F, Female; M, male; PA, pilocytic astrocytoma; GBM, glioblastoma; OD, oligodendroglioma; AA, anaplastic astrocytoma; GG, ganglioglioma; $B R A F$ : v-raf murine sarcoma viral oncogene homolog $\mathrm{B} 1$; +: fusion breakpoint detected.

\section{Results}

Patients. The clinical records of 19 patients (10 girls and 9 boys) with a mean age at surgery of 7.2 years (ranging from 1 month to 14 years) were retrospectively examined. At least two pathologists examined the tumour samples and reached a consensus on their diagnosis based on the World Health Organization Classification of Tumours of the Central Nervous System (1). Five types of paediatric brain tumours were studied: pilocytic astrocytoma, ganglioglioma, oligodendroglioma, anaplastic astrocytoma and glioblastoma (Table II). The details of the patients' data are summarised in Table III.

Detection of KIAA1549-BRAF fusions. KIAA1549-BRAF fusion and its breakpoints was successfully identified in all five types of tumour (Table II). Figure 1 depicts representative pyrograms showing their breakpoint sequences. The pyrogram clearly showed the three breakpoints sequences. KIAA1549-BRAF fusion was detected in all of the types of tumours examined. Among the 10 pilocytic astrocytomas, nine had KIAA-BRAF fusion (90\%). Three kinds of tumours (3 pilocytic astrocytoma, 1 glioblastoma, and 1 oligodendroglioma) had multiple fusions.

Time required for analysis. The entire procedure from RNA extraction to obtaining mutation data was performed in $2 \mathrm{~h}$ or less. The details of the procedure are summarised in Figure 2.

\section{Discussion}

Detection of the KIAA1549-BRAF fusion variant. The detection of KIAA1549-BRAF fusions with their specific breakpoints is important in clinical settings (13). The identification of the type of fusion present is important for prognosis; therefore, any diagnostic method implemented should provide this information (15). We achieved this using the Pyromark system. Not all previously reported studies on pilocytic astrocytoma mention the fusion gene variant. We found studies related to KIAA1549-BRAF fusions in pilocytic astrocytoma by searching PubMed using the search terms 'KIAA1549', 'BRAF', 'fusion gene' and 'pilocytic astrocytoma'. Searches were limited to human studies published in English from 2014 and 2017. We found 10 studies that demonstrated detection methods for KIAA1549-BRAF fusion (10, 14-22). Five of these studies identified the fusion gene variant using direct sequencing, and three studies used only FISH. The results of FISH are often difficult to interpret because it is difficult to distinguish a fusion signal from a normal one (23). As mentioned earlier, FISH cannot identify the fusion gene breakpoint; hence a phenotype cannot be assigned to a particular variant, and the information obtained from these studies is limited. Therefore, a method like the one we used here is superior because it helps assign clinical features to specific fusion genes. 

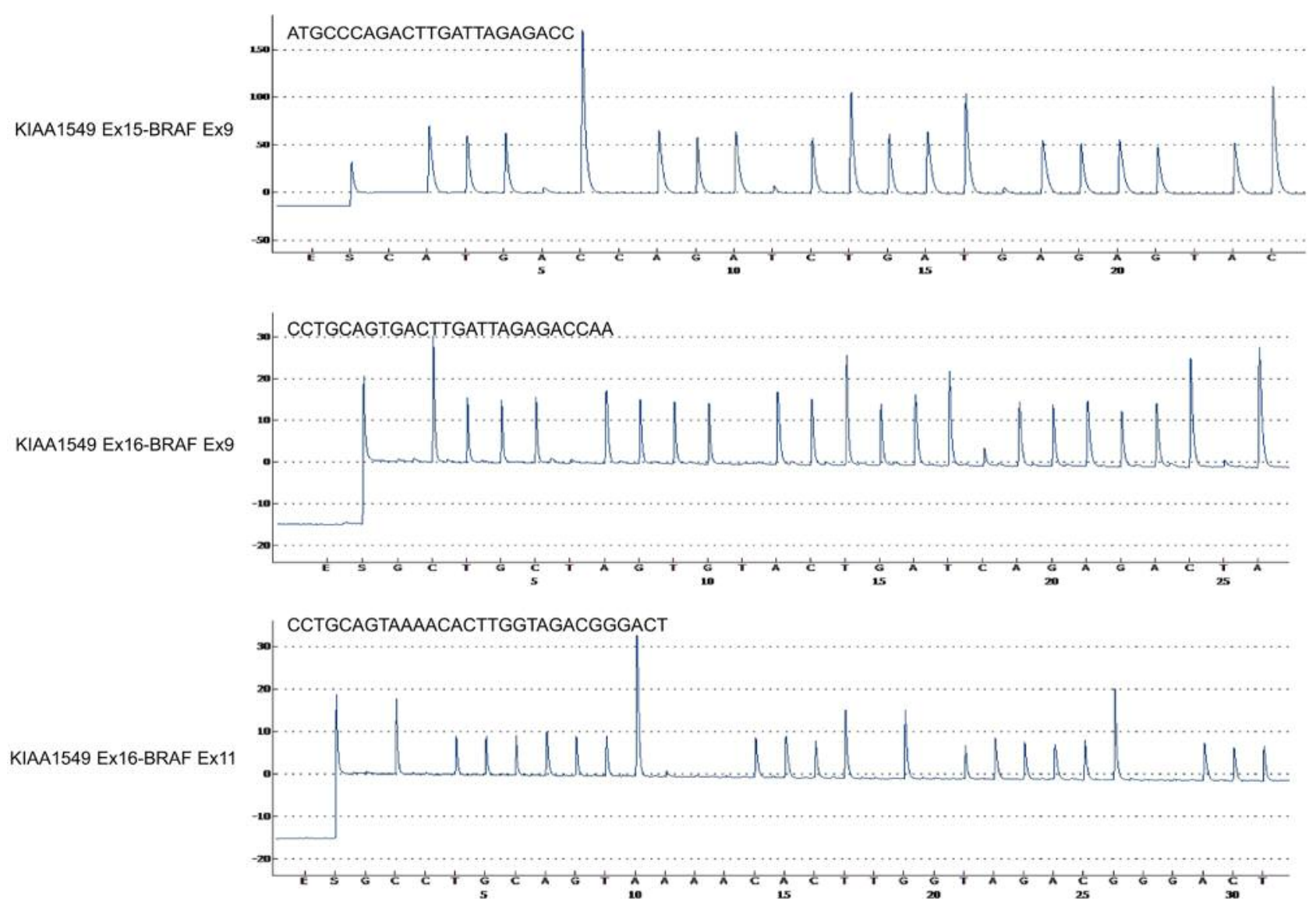

Figure 1. Representative pyrogram images showing the sequences as clearly identified strong signals.

KIAA1549-BRAF fusion in paediatric brain tumours. We successfully detected KIAA1549-BRAF fusion and its breakpoints in 14 out of 19 patients presenting five types of paediatric brain tumours. Few studies have investigated the relevance of KIAA1549-BRAF fusion in paediatric brain tumours other than those focussing on pilocytic astrocytomas. Especially in cases of high-grade glioma, the significance of KIAA1549-BRAF fusion has not been well investigated. Studies on 15 adult glioblastoma cases found no KIAA1549-BRAF fusions $(1,23)$. In another study, two out of 157 patients with high-grade gliomas under the age of 18 years had KIAA1549-BRAF fusions, and both had the same fusion breakpoint between exons 15 and 9 (24). Our study showed that two out of five patients with high-grade glioma, anaplastic astrocytoma and glioblastoma had KIAA1549-BRAF fusions; one of them with a fusion between exons 16 and 9 . To the best of our knowledge, this fusion breakpoint has not been previously reported in a glioblastoma. These important findings in our small-scale study show the potential of further application of our method that may be helpful even as an alternative to next-generation sequencing, which is considered accurate and sensitive for detecting different genetic alterations (25).

Time required for analysis. The commonly used methods for detecting fusion breakpoints involve direct sequencing following RT-PCR, and take at least $4 \mathrm{~h}$ to obtain results (60 min for the PCR run and 3-4 h for the cyclic amplification). Our method, on the other hand, required just $2 \mathrm{~h}$, indicating the diagnostic results can be made available to surgeons during the operation itself. In addition, the histopathological distinction between pilocytic astrocytoma and malignant glioma can be challenging because pilocytic astrocytomas are occasionally accompanied by necrosis and microvascular proliferation and may look like malignant gliomas, particularly in frozen sections examined during surgery. In cases in which the results of intraoperative histopathological diagnosis are ambiguous, intraoperative molecular diagnosis using our method may allow the surgeon to decide on the best surgical approach for the patient. 


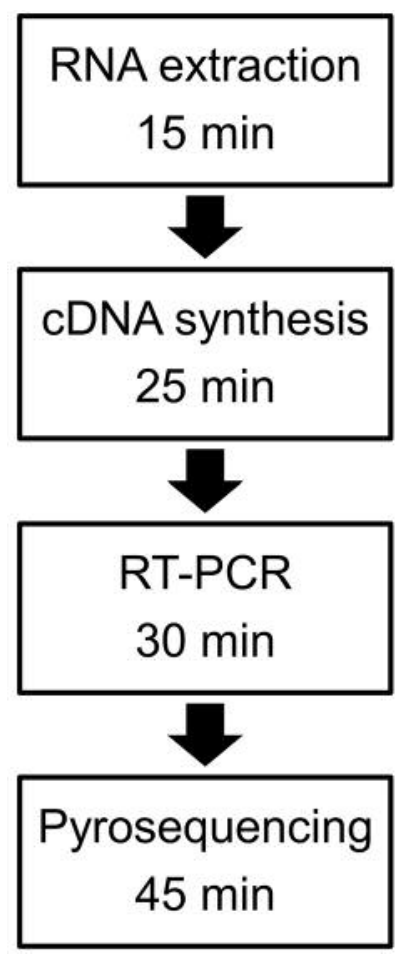

Figure 2. Flow chart of the KIAA1549 exon 16-v-raf murine sarcoma viral oncogene homolog B1 (BRAF) assay methodology.

Limitations of this method and study. Our method clearly showed the presence of fusion gene variants. However, we detected only the three most common fusion variants, and studies have reported that KIAA1549-BRAF fusions can involve different exons and fusions such as KIAA1549 exon15-BRAF exon-11 and KIAA1549 exon-17-BRAF exon-10 (14). Increasing the number of variants examined may increase the time needed to obtain results, and thus our method may not be suitable for detecting fusion genes with a large number of variants.

In addition, we studied only detection of variants of KIAA1549-BRAF fusion; therefore, we cannot account for the impact of different fusion genes. We found two cases of paediatric oligodendroglioma with KIAA1549-BRAF fusion. The presence of this type of oligodendroglioma may represent a subset of oligodendrogliomas sharing characteristics of pilocytic astrocytomas, particularly in children (14). Of note, activation of the mitogen-activated protein kinase pathway by KIAA1549-BRAF fusion in pilocytic astrocytomas has drawn the attention of paediatric neuro-oncologists because of its potential as a therapeutic target (26). Our study involved only a small number of samples, and we did not compare our methods with other detecting methods; in order to confirm the clinical implications using this method and generalise our findings to other tumours, further studies are necessary.

\section{Conclusion}

In this study, we used a comprehensive, quick method for the detection of KIAA1549-BRAF fusion from tumour tissues of paediatric patients with brain tumours. This method detected three KIAA1549-BRAF fusion variants within $2 \mathrm{~h}$ of collection of the tumour specimen. Our results will be useful for elucidating tumourigenesis of paediatric brain tumours and studies on intraoperative diagnosis and classification of tumours for improving surgical decisions.

\section{Acknowledgements}

The Authors thank the patients for their participation in this study. The Graduate School Research Program of Juntendo University and the Juntendo University Research Institute for Diseases of Old Age (Tokyo, Japan) supported the present study.

\section{References}

1 von Deimling A (eds): WHO classification of tumours of the central nervous system. IARC, Lyon, 2016.

2 Wijnenga MM, French PJ, Dubbink HJ, Dinjens WN, Atmodimedjo PN, Kros JM, Fleischeuer R, Dirven CM, Vincent AJ, van den Bent MJ: Prognostic relevance of mutations and copy number alterations assessed with targeted next generation sequencing in IDH mutant grade II glioma. J Neurooncol: 1-9, 2018.

3 Dupain C, Harttrampf AC, Urbinati G, Geoerger B and MassaadMassade L: Relevance of fusion genes in paediatric cancers: toward precision medicine. Mol Ther Nucleic Acids 6: 315-326, 2017.

4 Duesberg PH: Cancer genes generated by rare chromosomal rearrangements rather than activation of oncogenes. Haematol Blood Transfus 31: 496-510, 1987.

5 Yoshihara K, Wang Q, Torres-Garcia W and Zheng S, Vegesna R, Kim H and Verhaak RG: The landscape and therapeutic relevance of cancer-associated transcript fusions. Oncogene 34 : 4845-4854, 2015.

6 Selfe J, Olmos D, Al-Saadi R, Thway K, Chisholm J, Kelsey A and Shipley J: Impact of fusion gene status versus histology on risk-stratification for rhabdomyosarcoma: Retrospective analyses of patients on UK trials. Pediatr Blood Cancer 64, 2017. doi: $10.1002 / \mathrm{pbc} .26386$

7 Lin A, Rodriguez FJ, Karajannis MA, Williams SC, Legault G, Zagzag D, Burger PC, Allen JC, Eberhart CG and Bar EE: BRAF alterations in primary glial and glioneuronal neoplasms of the central nervous system with identification of 2 novel KIAA1549:BRAF fusion variants. J Neuropathol Exp Neurol 71: 66-72, 2012.

8 Tatevossian RG, Lawson AR, Forshew T, Hindley GF, Ellison DW and Sheer D: MAPK pathway activation and the origins of pediatric low-grade astrocytomas. J Cell Physiol 222: 509-514, 2010.

9 Yuzawa S, Nishihara H, Wang L, Tsuda M, Kimura T, Tanino M and Tanaka S: Analysis of NAB2-STAT6 gene fusion in 17 cases of meningeal solitary fibrous tumor/hemangiopericytoma: review of the literature. Am J Surg Pathol 40: 1031-1040, 2016. 
10 Hasselblatt M, Riesmeier B, Lechtape B, Brentrup A, Stummer W, Albert FK, Sepehrnia A, Ebel H, Gerss J and Paulus W: $B R A F-K I A A 1549$ fusion transcripts are less frequent in pilocytic astrocytomas diagnosed in adults. Neuropathol Appl Neurobiol 37: 803-806, 2011.

11 Von Deimling A, Korshunov A and Hartmann C: The next generation of glioma biomarkers: $M G M T$ methylation, BRAF fusions and IDH1 mutations. Brain Pathol 21: 74-87, 2011.

12 Setty P, Gessi M, Waha A, Hammes J, El-Maarri O and Pietsch $\mathrm{T}$ and Waha A: Sensitive determination of BRAF copy number in clinical samples by pyrosequencing. Diagn Mol Pathol 20 : 148-157, 2011.

13 Berghoff AS and Preusser M: BRAF alterations in brain tumours: molecular pathology and therapeutic opportunities. Curr Opin Neurol 27: 689-696, 2014.

14 Kumar A, Pathak P, Purkait S, Faruq M, Jha P, Mallick S, Suri V, Sharma MC, Suri A and Sarkar C: Oncogenic KIAA1549$B R A F$ fusion with activation of the MAPK/ERK pathway in pediatric oligodendrogliomas. Cancer Genet 208: 91-95, 2015.

15 Faulkner C, Ellis HP, Shaw A, Penman C, Palmer A, Wragg C, Greenslade M, Haynes HR, Williams H, Lowis S and White P: $B R A F$ fusion analysis in pilocytic astrocytomas: KIAA1549$B R A F$ 15-9 fusions are more frequent in the midline than within the cerebellum. J Neuropathol Exp Neurol 74: 867-872, 2015.

16 Roth JJ, Fierst TM, Waanders AJ, Yimei L, Biegel JA and Santi M: Whole chromosome 7 gain predicts higher risk of recurrence in pediatric pilocytic astrocytomas independently from KIAA1549-BRAF fusion status. J Neuropathol Exp Neurol 75: 306-315, 2016.

17 Antonelli M, Badiali M, Moi L, Buttarelli FR, Baldi C, Massimino M, Sanson M and Giangaspero F: KIAA1549:BRAF fusion gene in pediatric brain tumors of various histogenesis. Pediatr Blood Cancer 62: 724-727, 2015.

18 Becker AP, Scapulatempo-Neto C and Carloni AC, Paulino A, Sheren J, Aisner DL, Musselwhite E, Clara C, Machado HR, Oliveira RS and Neder L: KIAA1549: BRAF gene fusion and FGFR1 hotspot mutations are prognostic factors in pilocytic astrocytomas. J Neuropathol Exp Neurol 74: 743-754, 2015.

19 Taha H, Yehia M, Mahmoud M, El-Beltagy M and Ghabriel M and El-Naggar S: Incidence of KIAA1549-BRAF fusion gene in Egyptian pediatric low-grade glioma. Clin Transl Med 4: 10, 2015.
20 Roth JJ, Santi M, Pollock AN, Harding BN, Rorke-Adams LB, Tooke LS and Biegel JA: Chromosome band 7q34 deletions resulting in KIAA1549-BRAF and FAM131B-BRAF fusions in pediatric low-grade gliomas. Brain Pathol 25: 182-192, 2015.

21 Theeler BJ, Ellezam B, Sadighi ZS, Mehta V, Tran MD, Adesina AM, Bruner JM and Puduvalli VK: Adult pilocytic astrocytomas: clinical features and molecular analysis. Neuro Oncol 16: 841-847, 2014.

22 Cruz GR, Oliveira ID, Moraes L, Paniago MD, de Seixas Alves MT, Capellano AM, Saba-Silva N and Cavalheiro S, Cerutti JM and Toledo SR: Analysis of KIAA1549-BRAF fusion gene expression and $I D H 1 / I D H 2$ mutations in low grade pediatric astrocytomas. J Neurooncol 117: 235-242, 2014.

23 Tian Y, Rich BE, Vena N, Craig JM, MacConaill LE, Rajaram V, Goldman S, Taha H, Mahmoud M, Ozek M and Sav A: Detection of KIAA1549-BRAF fusion transcripts in formalinfixed paraffin-embedded pediatric low-grade gliomas. J Mol Diagn 13: 669-677, 2011.

24 Johnson A, Severson E, Gay L, Vergilio JA, Elvin J, Suh J, Daniel S, Covert M, Frampton GM, Hsu S and Lesser GJ: Comprehensive genomic profiling of 282 pediatric low-and high-grade gliomas reveals genomic drivers, tumor mutational burden, and hypermutation signatures. Oncologist 22: 14781490, 2017.

25 Nikiforova MN, Wald AI, Melan MA, Roy S, Zhong S, Hamilton RL, Lieberman FS, Drappatz J, Amankulor NM, Pollack IF and Nikiforov YE: Targeted next-generation sequencing panel (GlioSeq) provides comprehensive genetic profiling of central nervous system tumors. Neuro Oncol 18: 379-337, 2016.

26 Rodriguez FJ, Lim KS, Bowers D and Eberhart CG: Pathological and molecular advances in pediatric low-grade astrocytoma. Annu Rev Pathol 8: 361-379, 2013. 